
Preprints des Instituts für Mathematik der Universität Potsdam 

Preprints des Instituts für Mathematik der Universität Potsdam 5 (2016) 3

Dmitry Fedchenko | Nikolai Tarkhanov

Boundary Value Problems for Elliptic Complexes 
Bibliografische Information der Deutschen Nationalbibliothek

Die Deutsche Nationalbibliothek verzeichnet diese Publikation in der Deutschen Nationalbibliografie; detaillierte bibliografische Daten sind im Internet über http://dnb.dnb.de abrufbar.

\section{Universitätsverlag Potsdam 2016}

http://verlag.ub.uni-potsdam.de/

Am Neuen Palais 10, 14469 Potsdam

Tel.: +49 (0)331 9772533 / Fax: 2292

E-Mail: verlag@uni-potsdam.de

Die Schriftenreihe Preprints des Instituts für Mathematik der Universität Potsdam wird herausgegeben vom Institut für Mathematik der Universität Potsdam.

ISSN (online) 2193-6943

Kontakt:

Institut für Mathematik

Karl-Liebknecht-Straße 24/25

14476 Potsdam

Tel.: +49 (0)331977 1499

WWW: http://www.math.uni-potsdam.de

Titelabbildungen:

1. Karla Fritze | Institutsgebäude auf dem Campus Neues Palais

2. Nicolas Curien, Wendelin Werner | Random hyperbolic triangulation

Published at: http://arxiv.org/abs/1105.5089

Das Manuskript ist urheberrechtlich geschützt.

Online veröffentlicht auf dem Publikationsserver der Universität Potsdam

URL https://publishup.uni-potsdam.de/opus4-ubp/frontdoor/index/index/docId/8670

URN urn:nbn:de:kobv:517-opus4-86705

http://nbn-resolving.de/urn:nbn:de:kobv:517-opus4-86705 


\title{
BOUNDARY VALUE PROBLEMS FOR ELLIPTIC COMPLEXES
}

\author{
D. FEDCHENKO AND N. TARKHANOV
}

\begin{abstract}
The aim of this paper is to bring together two areas which are of great importance for the study of overdetermined boundary value problems. The first area is homological algebra which is the main tool in constructing the formal theory of overdetermined problems. And the second area is the global calculus of pseudodifferential operators which allows one to develop explicit analysis.
\end{abstract}

\section{Contents}

1. Introduction

2. Quotient complexes

3. Cauchy data relative to pseudodifferential operators

4. The complex of Cauchy data

5. Cone of a cochain mapping

6. A noncharacteristic Cauchy problem

7. The Cauchy problem for the Dolbeault complex

References

\section{IntRoduction}

The unquenchable phantasy of a researcher might pose any question on complexes of differential operators on a manifold with boundary. However, mathematics need not have any promising answers to those questions which are not well motivated within the framework of the rigorous model.

As the second author presented his DSc thesis devoted to overdetermined systems of differential equations in the Moscow state university in 1991, Y.S. Il'yashenko asked him about remarkable complexes which are different from the classical de Rham and Dolbeault complexes and their twisted versions. The author should acknowledge that there had perhaps been not many of them, see however [Tar90], [Kha14] and elsewhere.

Actually complexes of differential operators are naturally graded determined systems of scalar differential operators. The fundamental problem related to any system of partial differential equations is that on the solvability of the system. A boundary value problem for the system is an efficient way to parametrise the solutions through their boundary data. For complexes of differential operators a relevant substitute for a solution is the cohomology of the complex. Therefore, for

Date: October 25, 2015.

2010 Mathematics Subject Classification. Primary 58G03; Secondary 47A53, 35Jxx.

Key words and phrases. Elliptic complexes, Fredholm property, index. 
complexes those boundary value problems are of key interest which retain information on the cohomology.

Such a construction can be adopted immediately from homological algebra where it is called the cone of a cochain mapping, see [Spa66] and elsewhere. To the best of our knowledge the construction was first used in [Dyn72] to introduce elliptic boundary problems for complexes of pseudodifferential operators on a compact manifold with boundary.

The idea of [Dyn72] was corroborated in the paper [DS96] which established that under a nondegeneracy assumption any overdetermined boundary value problem possesses a compatibility complex of boundary value problems which bears the structure of the cone of a cochain mapping. Both [Dyn72] and [DS96] suffer from incomplete proofs of existence theory. This gap was filled up within well elaborated techniques in [KTT07].

In [KMST05] one applies the construction of the cone of a cochain mapping to get Fredholm boundary value problems for the Dolbeault complex on a compact complex manifold with boundary. As but one motivation of [KMST05] we mention that the Dolbeault complex on a strongly pseudoconvex compact manifold is subelliptic at positive steps. What has still been lacking in [KMST05] is the general construction of elliptic boundary value problems within a so-called Boutet de Monvel's algebra with Toeplitz boundary conditions, see [SS15]. Such a construction for complexes on compact manifolds with boundary elliptic with respect to the interior symbol is elaborated in the recent paper [SS15]. It appeals to techniques developed in [Tar07].

While the desire to describe a similar construction was the first aim of the present paper, on reflecting upon the problem we found that the general construction yields any surprising elliptic boundary value problems neither for the de Rham complex nor for the Dolbeault complex on a compact manifold with boundary. It is numerous applied problems that give an inexhaustible source of ample mathematical phenomena.

The boundary value problems for complexes we consider in the present paper are greatly motivated by [ST00]. When extremely simplified, they are traced back to the following classical problem of algebraic topology. Let $\mathcal{X}$ and $\mathcal{S}$ be two $C^{\infty}$ compact closed manifolds and $\iota: \mathcal{S} \rightarrow \mathcal{X}$ a differentiable mapping. The 'pull-back' $\iota^{*}$ under $\iota$ gives us a cochain mapping of the de Rham complex on $\mathcal{X}$ into that on $\mathcal{S}$, namely, $\iota^{*}: \Omega(\mathcal{X}) \rightarrow \Omega \cdot(S)$. The cone $\mathcal{C}\left(\iota^{*}\right)$ of this cochain mapping is said to be the cone of $\iota$. The complex $\mathcal{C}\left(\iota^{*}\right)$ is easily seen to be Fredholm, i.e., it bears a finitedimensional cohomology $H^{i}\left(\mathcal{C}\left(\iota^{*}\right)\right)$, for each $i$. Moreover, $H^{i}\left(\mathcal{C}\left(\iota^{*}\right)\right)$ is naturally isomorphic to the relative cohomology of the pair $(M, \iota(S))$ with coefficients in $\mathbb{C}$, provided that $\iota$ is an embedding. We now assume that $f$ is a smooth mapping of the pair $(\mathcal{X}, \mathcal{S})$, i.e., $f=\left(f_{\mathcal{X}}, f_{\mathcal{S}}\right)$ where $f_{\mathcal{X}}$ and $f_{\mathcal{S}}$ are smooth mappings of $\mathcal{X}$ and $\mathcal{S}$, respectively. If the diagram

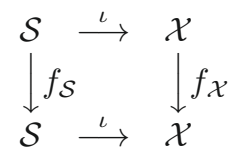

commutes, then $f$ has a natural lift to the complex $\mathcal{C}\left(\iota^{*}\right)$ given by $f_{\mathcal{X}}^{*} \oplus f_{\mathcal{S}}^{*}$. Denote by $L\left(f^{*}\right)$ the Lefschetz number of this endomorphism of $\mathcal{C}\left(\iota^{*}\right)$. By the above, in case $\iota$ is an embedding $L\left(f^{*}\right)$ coincides with the Lefschetz number in the relative 
cohomology of $(\mathcal{X}, \iota(\mathcal{S}))$ induced by $f_{\mathcal{X}}$. This latter is well-defined because $\iota(\mathcal{S})$ is invariant under the mapping $f_{\mathcal{X}}$. For the de Rham complex, our result reads $L\left(f^{*}\right)=L\left(f_{\mathcal{X}}^{*}\right)-L\left(f_{\mathcal{S}}^{*}\right)$ which does not explicitly involve $\iota$. More generally, we consider two elliptic complexes $C^{\infty}\left(\mathcal{X}, F^{*}\right)$ and $C^{\infty}\left(\mathcal{S}, E^{\cdot}\right)$ on $\mathcal{X}$ and $\mathcal{S}$, respectively. To each cochain mapping $T: C^{\infty}\left(\mathcal{X}, F^{\cdot}\right) \rightarrow C^{\infty}\left(\mathcal{S}, E^{\cdot}\right)$ there corresponds a new complex $\mathcal{C}(T)$ called the cone of $T$, cf. $i b i d$.. If $T$ is of finite order relative to the scales of Sobolev spaces on $\mathcal{X}$ and $\mathcal{S}$, then $\mathcal{C}(T)$ proves to be Fredholm. In parallel to the relative de Rham cohomology, the corresponding theory in the case of general elliptic complexes on $\mathcal{X}$ and $\mathcal{S}$ is referred to as the 'relative elliptic theory'. Yet another example of great importance in geometry is the cone of a holomorphic mapping of two complex manifolds $\iota: \mathcal{S} \rightarrow \mathcal{X}$. In this latter case both $C^{\infty}\left(\mathcal{X}, F^{\cdot}\right)$ and $C^{\infty}\left(\mathcal{S}, E^{*}\right)$ are the Dolbeault complexes on $\mathcal{X}$ and $\mathcal{S}$, respectively. The paper [ST00] contains a proof of the Lefschetz fixed point formula in the context of relative elliptic theory.

\section{Quotient COMPLEXeS}

Here we recall the well-known construction of a quotient complex from homological algebra (see [Spa66]).

Let $V^{\cdot}$ be a (cochain) complex of topological vector spaces whose differential is $d_{V}$. Suppose that for every $i \in \mathbb{Z}$ some subspace $U^{i}$ of $V^{i}$ is distinguished. If the differential $d_{V}$ preserves this sequence of subspaces, i.e. $d_{V} U^{i} \subset U^{i+1}$ for all $i$, then the sequence $U^{i}$ is a complex of topological vector spaces itself, endowed with the same differential $d_{V}$. As usual, such a complex $U$ is referred to as a subcomplex of $V$.

If $U^{\cdot}$ is a subcomplex of $V^{\cdot}$, we can construct a new complex of topological vector spaces $Q^{\cdot}$ in the following way. Set $Q^{i}=V^{i} / U^{i}$ for $i \in \mathbb{Z}$. The differential $d_{Q}$ is defined so as to make the next diagram, whose rows are exact, commutative

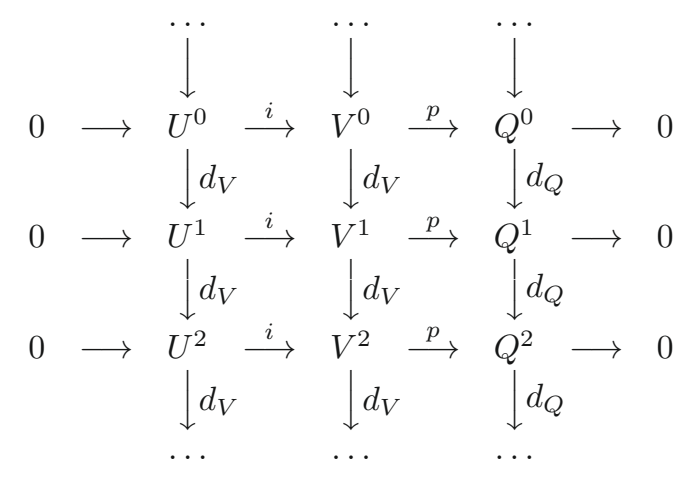

Here the mappings labeled $i$ represent inclusion and those labeled $p$ represent projection onto the quotient. It is easy to verify that $d_{Q}$ is continuous and $d_{Q}^{2}=0$. The complex $Q$ obtained this way is called the quotient complex of $V$ over $U^{\cdot}$ and it is denoted by $V^{\cdot} / U$.

\section{Cauchy data relative to pseudodifferential operators}

Assume that $\mathcal{X}$ be a compact $C^{\infty}$ manifold with boundary containing in a larger smooth manifold $\mathcal{X}^{\prime}$. Let $A$ be a pseudodifferential operator of order $m \in \mathbb{Z}$ acting between sections of vector bundles $E$ and $F$ over $\mathcal{X}^{\prime}$. If $u \in C^{\infty}(\mathcal{X}, E)$, then the 
D. FEDCHENKO AND N. TARKHANOV

extension $e^{+} u$ of $u$ to all of $\mathcal{X}^{\prime}$ by zero belongs to $L_{\text {comp }}^{2}\left(\mathcal{X}^{\prime}, E\right)$, and hence the image $A\left(e^{+} u\right)$ lies in $H_{\mathrm{loc}}^{-m}\left(\mathcal{X}^{\prime}, F\right)$. By the pseudolocality property of pseudodifferential operators the restriction $r^{+} A\left(e^{+} u\right)$ of $A\left(e^{+} u\right)$ to the interior of $\mathcal{X}$ is $C^{\infty}$. The operator $A$ is said to possess the transmission property with respect to the surface $\partial \mathcal{X}$ if $r^{+} A\left(e^{+} u\right)$ extends smoothly to the boundary of $\mathcal{X}$ whenever $u \in C^{\infty}(\mathcal{X}, E)$. For the resulting operator one writes simply $A: C^{\infty}(\mathcal{X}, E) \rightarrow C^{\infty}(\mathcal{X}, F)$. In the sequel we restrict ourselves to pseudodifferential operators with transmission property with respect to $\partial \mathcal{X}$. As but one example we mention partial differential operators on $\mathcal{X}$.

Denote by $A^{*}$ the formal adjoint operator for $A$. This is a pseudodifferential operator of the same order $m$ from sections of $F$ to sections of $E$ on $\mathcal{X}^{\prime}$, such that $(A u, g)_{\mathcal{X}^{\prime}}=\left(u, A^{*} g\right)_{\mathcal{X}^{\prime}}$ for all $u \in C_{\text {comp }}^{\infty}\left(\mathcal{X}^{\prime}, E\right)$ and $g \in C_{\text {comp }}^{\infty}\left(\mathcal{X}^{\prime}, F\right)$. Here, $(\cdot, \cdot) \mathcal{X}^{\prime}$ stands for the scalar product in $L^{2}(\mathcal{X}, F)$ or $L^{2}(\mathcal{X}, E)$, respectively. The formal adjoint operator bears the transmission property with respect to $\partial \mathcal{X}$ along with $A$.

A section $u \in C^{\infty}(\mathcal{X}, E)$ is said to have zero Cauchy data on $\partial \mathcal{X}$ relative to $A$ if $(A u, g)_{\mathcal{X}}=\left(u, A^{*} g\right)_{\mathcal{X}}$ for all $u \in C^{\infty}(\mathcal{X}, E)$ and $g \in C^{\infty}(\mathcal{X}, F)$. In this case we write $c_{A}(u)=0$.

Lemma 3.1. If $m \leq 0$, then each section $u \in C^{\infty}(\mathcal{X}, E)$ has zero Cauchy data on $\partial \mathcal{X}$ relative to $A$.

Proof. Let $g \in C^{\infty}(\mathcal{X}, F)$. Pick any sequence $g_{j} \in C^{\infty}\left(\mathcal{X}^{\prime}, F\right)$ with the property that the support of each $g_{j}$ belongs to the interior of $\mathcal{X}$ and $g_{j} \rightarrow g$ in the norm of $L^{2}(\mathcal{X}, F)$. Since $A u=r^{+} A\left(e^{+} u\right)$ belongs to $L^{2}(\mathcal{X}, F)$, we conclude by the continuity that

$$
\begin{aligned}
(A u, g)_{\mathcal{X}} & =\lim _{j \rightarrow \infty}\left(A u, g_{j}\right)_{\mathcal{X}} \\
& =\lim _{j \rightarrow \infty}\left(A\left(e^{+} u\right), g_{j}\right)_{\mathcal{X}^{\prime}} \\
& =\lim _{j \rightarrow \infty}\left(e^{+} u, A^{*} g_{j}\right)_{\mathcal{X}^{\prime}} \\
& =\left(u, A^{*} g\right)_{\mathcal{X}},
\end{aligned}
$$

as desired.

For partial differential operators $A$ the following theorem is easily obtained by partial integration.

Theorem 3.2. Suppose $m>0$. If $u \in C^{\infty}(\mathcal{X}, E)$ vanishes up to order $m-1$ on $\partial \mathcal{X}$, then $u$ has zero Cauchy data on $\partial \mathcal{X}$.

Proof. Let $u \in C^{\infty}(\mathcal{X}, E)$ vanish up to the order $m-1$ on the boundary of $\mathcal{X}$, i.e., $\partial^{\alpha} u=0$ on $\partial \mathcal{X}$ for all multi-indices $\alpha$ satisfying $|\alpha| \leq m-1$. By the spectral synthesis theorem, there is a sequence $u_{j} \in C^{\infty}(\mathcal{X}, E)$, such that each $u_{j}$ is compactly supported in the interior of $\mathcal{X}$ and $u_{j} \rightarrow u$ in the norm of $H^{m}(\mathcal{X}, E)$. If $g \in C^{\infty}(\mathcal{X}, F)$, then $A^{*}\left(e^{+} g\right) \in H_{\mathrm{loc}}^{-m}\left(\mathcal{X}^{\prime}, E\right)$ which is the dual space of $H_{\mathrm{comp}}^{m}\left(\mathcal{X}^{\prime}, E\right)$. Therefore, we get

$$
\begin{aligned}
(A u, g)_{\mathcal{X}} & =\lim _{j \rightarrow \infty}\left(A u_{j}, g\right)_{\mathcal{X}} \\
& =\lim _{j \rightarrow \infty}\left(u_{j}, r^{+} A^{*}\left(e^{+} g\right)\right)_{\mathcal{X}^{\prime}} \\
& =\left(u, A^{*} g\right)_{\mathcal{X}},
\end{aligned}
$$


as desired.

Denote by $C_{c_{A}}^{\infty}(\mathcal{X}, E)$ the subspace of $C^{\infty}(\mathcal{X}, E)$ consisting of those sections $u$ whose Cauchy data on the boundary relative to $A$ vanish. Obviously, this subspace is closed. On combining Lemma 3.1 and Theorem 3.2 we conclude that the quotient space

$$
Q=\frac{C^{\infty}(\mathcal{X}, E)}{C_{C_{A}}^{\infty}(\mathcal{X}, E)}
$$

is zero, if $m \leq 0$, and it is specified within $C^{\infty}\left(\partial \mathcal{X}, J^{m-1}(E)\right)$, if $m \geq 1$. Here, $J^{m-1}(E)$ stands for the bundle of $(m-1)$-jets of smooth sections of the bundle $E$ over $\mathcal{X}^{\prime}$.

The quotient space $Q$ bears the Cauchy data of sections $u \in C^{\infty}(\mathcal{X}, E)$ on the surface $\partial \mathcal{X}$ relative to $A$. This leads immediately to a Green formula for the pseudodifferential operator $A$ on the manifold with boundary $\mathcal{X}$, cf. [See66]. The main difficulty in carrying out this construction for pseudodifferential operators is that the spaces of Cauchy data do not survive under the action of the differential of a complex. Hence, the spaces of Cauchy data do not fit well into the constructions of the cone of a cochain mapping of homological algebra, see [Spa66]. We restrict ourselves to those complexes whose differentials are given by local (i.e., differential) operators, in which case the methods of homological algebra yield useful calculating tools.

\section{The complex of Cauchy data}

For $i=0,1, \ldots, N-1$, let $A^{i}$ be a differential operator of order $m_{i}$ between sections of vector bundles $F^{i}$ and $F^{i+1}$ on $\mathcal{X}^{\prime}$. Assume that the composition of any two successive operators of the sequence is zero, and consider the complex of Fréchet spaces

$$
0 \longrightarrow C^{\infty}\left(\mathcal{X}, F^{0}\right) \stackrel{A}{\longrightarrow} C^{\infty}\left(\mathcal{X}, F^{1}\right) \stackrel{A}{\longrightarrow} \ldots \stackrel{A}{\longrightarrow} C^{\infty}\left(\mathcal{X}, F^{N}\right) \longrightarrow 0
$$

whose differential $A$ is given by $A u=A^{i} u$ for $u \in C^{\infty}\left(\mathcal{X}, F^{i}\right)$. It is convenient to set $A^{-1}=A^{N}=0$.

Lemma 4.1. If $u \in C^{\infty}\left(\mathcal{X}, F^{i}\right)$ has zero Cauchy data on $\partial \mathcal{X}$ relative to $A^{i}$, then also $c_{A}(A u)=0$.

Proof. Suppose that $u \in C^{\infty}\left(\mathcal{X}, F^{i}\right)$ satisfies $c_{A}(u)$ on $\partial \mathcal{X}$. By definition, this means that

$$
\left(A u, A^{*} g\right)_{\mathcal{X}}=\left(u,\left(A^{*}\right)^{2} g\right)_{\mathcal{X}}=\left(u,\left(A^{2}\right)^{*} g\right)_{\mathcal{X}}=0
$$

for all $g \in C^{\infty}\left(\mathcal{X}, F^{i+2}\right)$. On the other hand, we get $(A(A u), g)_{\mathcal{X}}=0$, for $A^{2}=0$. This implies the desired assertion.

The lemma shows that the differential $A$ of the complex $C^{\infty}\left(\mathcal{X}, F^{*}\right)$ preserves the spaces $C_{c_{A}}^{\infty}\left(\mathcal{X}, F^{\cdot}\right)$ of sections with zero Cauchy data. On using the construction of Section 2 we get the quotient complex

$$
Q=\frac{C^{\infty}\left(\mathcal{X}, F^{\cdot}\right)}{C_{c_{A}}^{\infty}\left(\mathcal{X}, F^{\cdot}\right)}
$$


whose differential is defined so as to make the following diagram with exact rows commutative

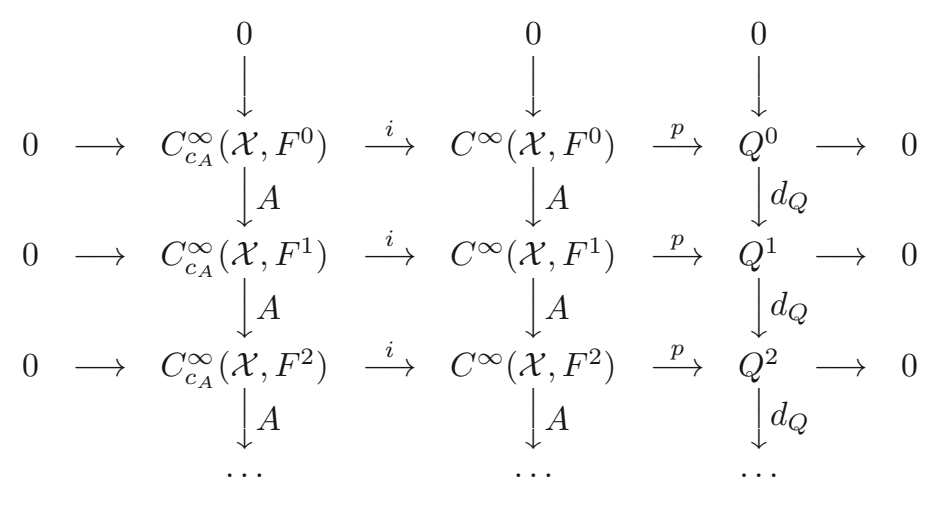

Here the mappings labeled $i$ represent inclusion and those labeled $p$ represent projection onto the quotient.

Recall that the hypersurface $\partial \mathcal{X}$ is said to be noncharacteristic for complex (4.3) at a point $x \in \partial \mathcal{X}$ if

$$
0 \longrightarrow F_{x}^{0} \stackrel{\sigma^{0}(x)}{\longrightarrow} F_{x}^{1} \stackrel{\sigma^{1}(x)}{\longrightarrow} \ldots \stackrel{\sigma^{N-1}(x)}{\longrightarrow} F_{x}^{N} \longrightarrow 0
$$

is an exact sequence of finite-dimensional vector spaces, where $F_{x}^{i}$ is the fibre of $F^{i}$ over the point $x$ and $\sigma^{i}(x)=\sigma^{m_{i}}\left(A^{i}\right)(x, d \varrho(x))$ is the principal symbol of $A^{i}$ evaluated at the cotangent vector $d \varrho(x)$. Here, $\varrho$ is a smooth real-valued function on $\mathcal{X}^{\prime}$, such that $\{\varrho<0\}$ just amounts to the interior of $\mathcal{X}$ and $\varrho^{\prime} \neq 0$ on the boundary of $\mathcal{X}$.

It was proved in [AN80] that if the boundary of $\mathcal{X}$ is noncharacteristic for complex (4.3) at each point, then the quotient complex $Q^{*}$ can be specified as a complex of differential operators $C^{\infty}\left(\partial \mathcal{X}, E^{*}\right)$ on $\partial \mathcal{X}$. This complex is often referred to as the tangential complex for (4.3), its differential is denoted by $A_{b}$ and the vector bundles $E^{i}$ by $F_{t}^{i}$, cf. [Tar90, $\left.\S 12\right]$. The construction of the tangential complex is traced back remarkably to classical complexes if $C^{\infty}\left(\mathcal{X}, F^{\cdot}\right)$ is a complex of first order differential operators, see [Tar90, 12.1]. We just mention that the tangential complex for the de Rham complex on $\mathcal{X}$ reduces to the de Rham complex on the closed manifold $\partial \mathcal{X}$.

\section{Cone of A COCHAin MAPPing}

Let

$$
\begin{aligned}
& 0 \longrightarrow V^{0} \stackrel{A}{\rightarrow} V^{1} \stackrel{A}{\longrightarrow} \ldots \stackrel{A}{\longrightarrow} V^{N} \rightarrow 0 \text {, } \\
& 0 \longrightarrow Q^{0} \stackrel{B}{\longrightarrow} Q^{1} \stackrel{B}{\longrightarrow} \ldots \stackrel{B}{\longrightarrow} Q^{N} \longrightarrow 0
\end{aligned}
$$

be two complexes of Banach spaces. By a cochain mapping of these complexes is meant any sequence of mappings

$$
C^{i}: V^{i} \rightarrow Q^{i}
$$


such that the diagram

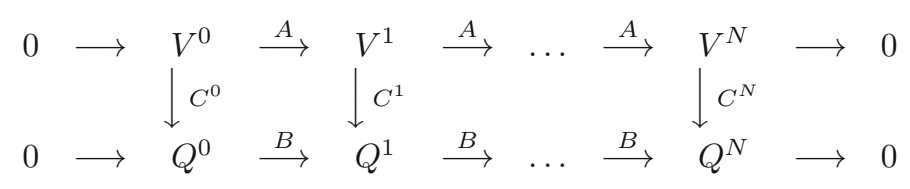

commutes, i.e., $C^{i} A=B C^{i-1}$ for all $i$.

Given any cochain mapping $C=\left\{C^{i}\right\}$, one defines a new complex

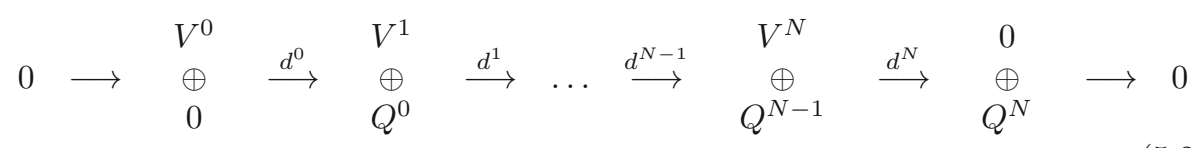

where

$$
d^{i}=\left(\begin{array}{cc}
-A^{i} & 0 \\
C^{i} & B^{i-1}
\end{array}\right)
$$

It is called the cone of the cochain mapping $C$ and denoted by $\mathcal{C}(C)$, cf. [Spa66] and elsewhere.

By $C^{i} A=B C^{i-1}$ we see that $A^{i}$ restricts to a mapping $\operatorname{ker} C^{i} \rightarrow \operatorname{ker} C^{i+1}$, and $B^{i}$ lifts to a mapping coker $C^{i} \rightarrow \operatorname{coker} C^{i+1}$. We thus arrive at two associated complexes

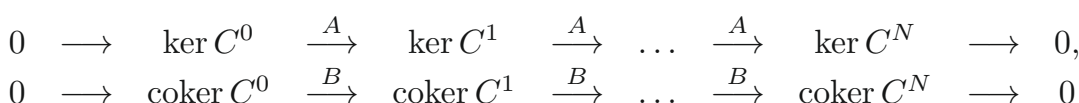

denoted by $\operatorname{ker} C$ and coker $C$, respectively.

Lemma 5.1. Complex (5.2) is Fredholm if and only if so are both the complexes (5.3). In this case

$$
\chi(\mathcal{C}(C))=\chi(\operatorname{ker} C)-\chi(\operatorname{coker} C) .
$$

Given a (cochain) complex $V^{*}$, we write $\chi\left(V^{*}\right)$ for the Euler characteristic of $V^{*}$, i.e, for the alternating sum of dimensions of the cohomology spaces of $V$, if defined.

Proof. A trivial verification shows that

$$
\begin{aligned}
H^{0}(\mathcal{C}(C)) & =H^{0}(\operatorname{ker} C), \\
H^{i}(\mathcal{C}(C)) & =H^{i}(\operatorname{ker} C) \oplus H^{i-1}(\operatorname{coker} C), \\
H^{N+1}(\mathcal{C}(C)) & =H^{N}(\operatorname{coker} C),
\end{aligned}
$$

and the lemma follows.

In particular, complex (5.2) is exact if and only if so are both the complexes

We apply the construction of $\mathcal{C}(C)$ in the case when

$$
Q^{i}=V^{i} / U^{i}
$$

$U^{i}$ being closed subspaces of $V^{i}$, such that $A$ maps $U^{i}$ to $U^{i+1}$ for each $i$. Then $A$ lifts in a natural way to a mapping $Q^{i} \rightarrow Q^{i+1}$ which we denote by $A_{Q}$. By 
the very definition it fulfills $C^{i} A=A_{Q} C^{i-1}$ where $C^{i}$ are the canonical mappings. Hence we arrive at the complex

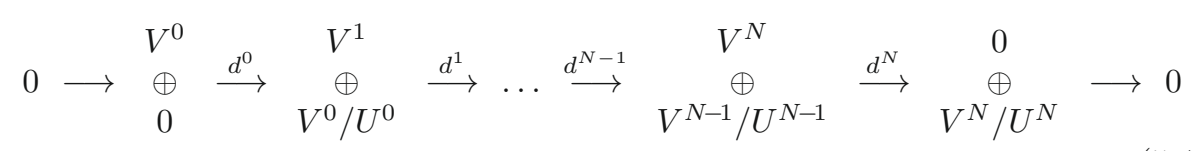

with

$$
d^{i}=\left(\begin{array}{rc}
-A^{i} & 0 \\
C^{i} & A_{Q}^{i-1}
\end{array}\right)
$$

Theorem 5.2. For complex (5.4) to be Fredholm it is necessary and sufficient that the complex

$$
0 \longrightarrow U^{0} \stackrel{A}{\longrightarrow} U^{1} \stackrel{A}{\longrightarrow} \ldots \stackrel{A}{\longrightarrow} U^{N} \rightarrow 0
$$

would be Fredholm. In this case the Euler characteristic of (5.4) is equal to that of the complex (5.5).

Proof. It suffices to combine Lemma 5.1 with the observation that in our case the complex $\operatorname{ker} C$ reduces to (5.5), and the complex coker $C$ is zero.

\section{A NONCHARACTERISTIC CAUChy PROBlem}

Suppose that the boundary of $\mathcal{X}$ is noncharacteristic for complex (4.3) at each point $x \in \partial \mathcal{X}$. Given $f \in C^{\infty}\left(\mathcal{X}, F^{i}\right)$ and $g \in C^{\infty}\left(\partial \mathcal{X}, F_{t}^{i-1}\right)$, we consider the Cauchy problem of finding sections $u \in C^{\infty}\left(\mathcal{X}, F^{i-1}\right)$ and $v \in C^{\infty}\left(\partial \mathcal{X}, F_{t}^{i-2}\right)$ which satisfy

$$
\left\{\begin{aligned}
-A u & =f \text { in } \mathcal{X} \\
c_{A}(u)+A_{b} v & =g \text { on } \partial \mathcal{X}
\end{aligned}\right.
$$

cf. [Tar90, § 19].

For the existence of a solution to this problem it is necessary that $-A f=0$ in $\mathcal{X}$ and $c_{A}(f)+A_{b} g=0$ on $\partial \mathcal{X}$. Hence, the study of problem (6.1) reduces to evaluating the cohomology of the complex

$$
\begin{array}{ccccc}
C^{\infty}\left(\mathcal{X}, F^{i-1}\right) & & C^{\infty}\left(\mathcal{X}, F^{i}\right) & & C^{\infty}\left(\mathcal{X}, F^{i+1}\right) \\
\oplus & \stackrel{d^{i-1}}{\longrightarrow} & \oplus & \stackrel{d^{i}}{\longrightarrow} & \oplus \\
C^{\infty}\left(\partial \mathcal{X}, F_{t}^{i-2}\right) & & C^{\infty}\left(\partial \mathcal{X}, F_{t}^{i-1}\right) & & C^{\infty}\left(\partial \mathcal{X}, F_{t}^{i}\right)
\end{array}
$$

at the middle step, where

$$
d^{i}=\left(\begin{array}{cc}
-A^{i} & 0 \\
c_{A} & A_{b}^{i-1}
\end{array}\right)
$$

The following example has been mentioned in many researches. It goes back at least as far as [Dyn72].

Example 6.1. Let $C^{\infty}\left(\mathcal{X}, F^{*}\right)=\Omega(\mathcal{X})$ be the de Rham complex on $\mathcal{X}$. Then (6.2) is the relative de Rham complex of the pair $(\mathcal{X}, \partial \mathcal{X})$ at step $i$. Its cohomology is isomorphic to the relative cohomology of the pair $(\mathcal{X}, \partial \mathcal{X})$ with complex coefficients, i.e., $H^{i}((\mathcal{X}, \partial \mathcal{X}), \mathbb{C})$. Hence, the cone of the cochain mapping $c_{d}$ of $\Omega(\mathcal{X})$ is Fredholm. 
For $i=0,1, \ldots, N$, we denote by $C_{\mathcal{X}}^{\infty}\left(\mathcal{X}^{\prime}, F^{i}\right)$ the subspace of $C^{\infty}\left(\mathcal{X}^{\prime}, F^{i}\right)$ consisting of all sections with (compact) support in $\mathcal{X}$. Obviously, the spaces $C_{\mathcal{X}}^{\infty}\left(\mathcal{X}^{\prime}, F^{i}\right)$ survive under the differential $A$ and so they constitute a subcomplex of $C^{\infty}\left(\mathcal{X}, F^{\cdot}\right)$.

Theorem 6.2. The cohomology of (6.2) at the middle step is isomorphic to that of $C_{\mathcal{X}}^{\infty}\left(\mathcal{X}^{\prime}, F^{\cdot}\right)$ at step i, i.e.,

$$
H^{i}\left(\mathcal{C}\left(c_{A}\right)\right) \equiv H^{i}\left(C_{\mathcal{X}}^{\infty}\left(\mathcal{X}^{\prime}, F^{\cdot}\right)\right)
$$

Proof. Since the boundary of $\mathcal{X}$ is noncharacteristic for complex (4.3) at each point, Theorem 11.11 of [Tar90] shows that the cohomology of the complex $C_{\mathcal{X}}^{\infty}\left(\mathcal{X}^{\prime}, F^{\cdot}\right)$ coincides with that of $C_{c_{a}}^{\infty}\left(\mathcal{X}, F^{\cdot}\right)$. Therefore, we shall have established the theorem if we prove that

$$
H^{i}\left(\mathcal{C}\left(c_{A}\right)\right) \equiv H^{i}\left(C_{c_{A}}^{\infty}\left(\mathcal{X}, F^{\cdot}\right)\right)
$$

Pick a cohomology class $[f \oplus g]$ in $H^{i}\left(\mathcal{C}\left(c_{A}\right)\right)$, where sections $f \in C^{\infty}\left(\mathcal{X}, F^{i}\right)$ and $g \in C^{\infty}\left(\partial \mathcal{X}, F_{t}^{i-1}\right)$ satisfy $-A f=0$ in $\mathcal{X}$ and $c_{A}(f)+A_{b} g=0$ on $\partial \mathcal{X}$. There is a section $\tilde{g} \in C^{\infty}\left(\mathcal{X}, F^{i-1}\right)$, such that $c_{A}(\tilde{g})=g$ on the boundary. Hence it follows that $A(f+A \tilde{g})=0$ in $\mathcal{X}$ and $c_{A}(f+A \tilde{g})=0$ on $\partial \mathcal{X}$. We introduce a homomorphism

$$
h: H^{i}\left(\mathcal{C}\left(c_{A}\right)\right) \rightarrow H^{i}\left(C_{c_{A}}^{\infty}\left(\mathcal{X}, F^{\cdot}\right)\right)
$$

by setting $h[f \oplus g]=[f+A \tilde{g}]$, the cohomology class in $H^{i}\left(C_{c_{A}}^{\infty}\left(\mathcal{X}, F^{\cdot}\right)\right)$ containing $f+A \tilde{g}$.

We first show that this definition is correct, i.e., the class $[f+A \tilde{g}]$ does not depend on the particular choice of the representative of the class $[f \oplus g]$. To this end, we assume that $f \oplus g$ represents the zero class in $H^{i}\left(\mathcal{C}\left(c_{A}\right)\right)$. Then we get $f=-A u$ in $\mathcal{X}$ and $g=c_{A}(u)+A_{b} v$ on $\partial \mathcal{X}$ for some sections $u \in C^{\infty}\left(\mathcal{X}, F^{i-1}\right)$ and $v \in C^{\infty}\left(\partial \mathcal{X}, F_{t}^{i-2}\right)$. Choose a section $\tilde{v} \in C^{\infty}\left(\mathcal{X}, F^{i-2}\right)$, such that $c_{A}(\tilde{v})=v$ on $\partial \mathcal{X}$. On setting $\tilde{g}=u+A \tilde{v}$ we obtain $c_{A}(u+A \tilde{v})=g$ and $[f+A \tilde{g}]=[f+A u]=0$, as desired.

The homomorphism $h$ is injective. Let $[f \oplus g]$ be any class in $H^{i}\left(\mathcal{C}\left(c_{A}\right)\right)$, such that $h[f \oplus g]=0$. By the definition of $h$, there is a section $u \in C^{\infty}\left(\mathcal{X}, F^{i-1}\right)$, such that $c_{A}(u)=0$ and $A u=f+A \tilde{g}$, where $\tilde{g} \in C^{\infty}\left(\mathcal{X}, F^{i-1}\right)$ satisfies $c_{A}(\tilde{g})=g$ on the boundary of $\mathcal{X}$. Then the section $-u+\tilde{g}$ belongs to $C^{\infty}\left(\mathcal{X}, F^{i-1}\right)$ and fulfills $-A(-u+\tilde{g})=f$ in $\mathcal{X}$ and $c_{A}(-u+\tilde{g})+A_{b} 0=g$. Hence it follows that $[f \oplus g]=0$, as desired.

It remains to show that the homomorphism $h$ is surjective. For this purpose, we fix a class $[f]$ in $H^{i}\left(C_{c_{A}}^{\infty}\left(\mathcal{X}, F^{i}\right)\right)$, where $f \in C^{\infty}\left(\mathcal{X}, F^{i}\right)$ is a section with the property that $A f=0$ in $\mathcal{X}$ and $c_{A}(f)=0$ on $\partial \mathcal{X}$. Then the pair $f \oplus 0$ determines obviously a cohomology class in $H^{i}\left(\mathcal{C}\left(c_{A}\right)\right)$ and $h[f \oplus 0]=[f+A 0]$, which completes the proof.

Actually, the abstract contents of the proof of this theorem is a part of homological algebra sketched in the proof of Lemma 5.1, for the cochain mapping $c_{A}$ is surjective.

Theorem 6.2 initiates many substantial problems of geometry and global analysis. Assume, e.g., that (4.3) is an elliptic complex on the manifold $\mathcal{X}^{\prime}$. Then it has a parametrix $P$ on $\mathcal{X}^{\prime}$, i.e., there is a sequence of pseudodifferential operators $P^{i}$ of order $-m_{i-1}$ between sections of vector bundles $F^{i}$ and $F^{i-1}$, such that $P A u+A P u=u-S u$ for all $u \in \mathcal{D}^{\prime}\left(\mathcal{X}^{\prime}, F^{i}\right)$ with compact support, where $S^{i}$ is 
a smoothing operator on sections of $\mathcal{D}^{\prime}\left(\mathcal{X}^{\prime}, F^{i}\right)$. Let now $u \in C_{\mathcal{X}}^{\infty}\left(\mathcal{X}^{\prime}, F^{0}\right)$ satisfy $A u=0$ in $\mathcal{X}^{\prime}$. Then $u=S u$ in $\mathcal{X}^{\prime}$. A familiar argument shows readily that the space of such $u$ is of finite dimension, i.e., $\operatorname{dim} H^{0}\left(C_{\mathcal{X}}^{\infty}\left(\mathcal{X}^{\prime}, F^{\cdot}\right)\right)<\infty$. On the other hand, suppose that $H^{1}\left(C^{\infty}\left(\mathcal{X}^{\prime}, F^{\cdot}\right)\right)=0$ and the complement of $\mathcal{X}$ in $\mathcal{X}^{\prime}$ is connected. Let $f \in C_{\mathcal{X}}^{\infty}\left(\mathcal{X}^{\prime}, F^{1}\right)$ satisfy $A f=0$ in $\mathcal{X}^{\prime}$. By assumption, there is a $u \in C^{\infty}\left(\mathcal{X}^{\prime}, F^{0}\right)$, such that $A u=f$ in $\mathcal{X}^{\prime}$. In particular, we get $A u=0$ away from the compact set $\mathcal{X}$ in $\mathcal{X}^{\prime}$. By the Hartogs theorem on removability of compact singularities by solutions of overdetermined systems (see [Tar90, $\S 19]$ ) we conclude that there is a section $\tilde{u} \in C^{\infty}\left(\mathcal{X}^{\prime}, F^{0}\right)$ satisfying $A \tilde{u}=0$ in $\mathcal{X}^{\prime}$ and $\tilde{u}=u$ in $\mathcal{X}^{\prime} \backslash \mathcal{X}$. The difference $u-\tilde{u} \in C^{\infty}\left(\mathcal{X}^{\prime}, F^{0}\right)$ is supported in $\mathcal{X}$ and it satisfies $A(u-\tilde{u})=f$ in $\mathcal{X}^{\prime}$. Hence it follows immediately that the cohomology $H^{1}\left(C_{\mathcal{X}}^{\infty}\left(\mathcal{X}^{\prime}, F^{\cdot}\right)\right)$ is trivial. It is worth pointing out that the cohomology of complex $C_{\mathcal{X}}^{\infty}\left(\mathcal{X}^{\prime}, F^{\cdot}\right)$ at the last step $N$ need not be finite-dimensional in general. A calculus of pseudodifferential operators relevant to the study of the cohomology of complex $C_{\mathcal{X}}^{\infty}\left(\mathcal{X}^{\prime}, F^{\cdot}\right)$ has been developed in [VE65].

\section{The Cauchy problem for the Dolbeault Complex}

Consider a compact domain $\mathcal{X}$ with Lipschitz boundary in an $n$-dimensional Kähler manifold $\left\{\mathcal{X}^{\prime}, \omega\right\}, \omega$ being a Kähler metric on $\mathcal{X}^{\prime}$. As usual, we write $\mathcal{X}^{\circ}$ for the interior of $\mathcal{X}$. We assume that $\mathcal{X}^{\circ}$ satisfies some $\log \delta$-pseudoconvexity condition, where $\delta$ stands for the boundary distance with respect to $\omega$. By the $\log \delta$ pseudoconvexity is meant roughly that $-\log \delta$ extends to a strictly plurisubharmonic function on $\mathcal{X}^{\circ}$. Examples of domains satisfying the above $\log \delta$-pseudoconvexity condition are weakly pseudoconvex domains in Stein manifolds and weakly pseudoconvex domains in Kähler manifolds with positive holomorphic bisectional curvature.

Let $f$ be a smooth $(p, q)$-form on $\mathcal{X}^{\prime}$ satisfying $\bar{\partial} f=0$ on $\mathcal{X}^{\prime}$ and $\operatorname{supp} f \subset \mathcal{X}$, i.e., $f$ vanishes to infinite order at the boundary of $\mathcal{X}$. The paper [Bri04] studies the problem of finding a smooth $(p, q-1)$-form $u$ on $\mathcal{X}^{\prime}$ with support in $\mathcal{X}$ satisfying $\bar{\partial} u=f$ in $\mathcal{X}^{\prime}$.

The solvability of this $\bar{\partial}$-problem leads to extension results for $\bar{\partial}_{b}$-closed forms on the boundary of $\mathcal{X}$, whenever $\partial \mathcal{X}$ is smooth, and can thus be used to understand the $\bar{\partial}_{b}$-cohomology of $\partial \mathcal{X}$, see [HN06] and [BH08] for a recent account of the theory. In [Bri04] it is proved that this $\bar{\partial}$-problem admits a solution for $1 \leq q \leq n-1$, and the top degree $\bar{\partial}$-cohomology groups of smooth forms with support in $\mathcal{X}$ are separated.

The case $\mathcal{X} \subset \mathbb{C}^{n}$ with piecewise smooth boundary was settled in the paper [MS99] using kernel methods. Note that a much more refined result based on explicit integral representations had already been established by Sh. Dautov in 1979 (see $\S 25$ in [AY79]).

Similarly to $[\mathrm{AY} 79, \S 25]$ the proof of [Bri04] exploits basic $L^{2}$ estimates on $\mathcal{X}$ with powers of the reciprocal of the boundary distance as weight function. Sobolev estimates for elliptic operators whose symbol can be controlled by some power of the boundary distance are deduced in order to prove regularity results for the minimal $L^{2}$ solutions of the $\bar{\partial}$-equation. 
Theorem 6.2 allows one to apply the results of [Bri04] to the cone of the cochain mapping

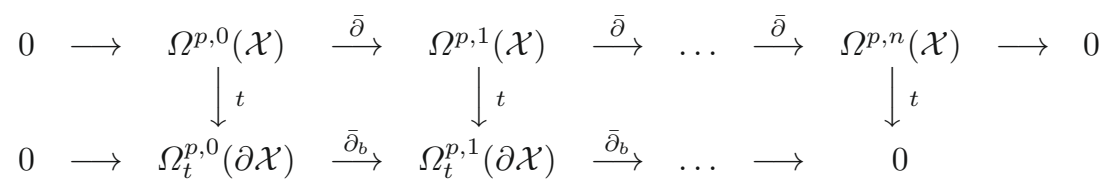

of the Dolbeault complex $\Omega^{p, \cdot}(\mathcal{X})$ on $\mathcal{X}$ into the induced complex $\Omega_{t}^{p, \cdot}(\partial \mathcal{X})$ on the boundary, see [Tar90, $\S 11]$. Here, for fixed $p=0,1, \ldots, n$, by $\Omega^{p, q}(\mathcal{X})$ is meant the space of all differential forms of bidegree $(p, q)$ with $C^{\infty}$ "coefficients" on $\mathcal{X}$. Given any $f \in \Omega^{p, q}(\mathcal{X})$, we denote by $t(f)$ the complex tangential part of $f$ on $\partial \mathcal{X}$. As mentioned, $t(f)$ is identified as a $C^{\infty}$ section of a smooth vector bundle over the boundary which is different from zero unless $q=n$. The space of all such sections is denoted by $\Omega_{t}^{p, q}(\partial \mathcal{X})$, for $q=0,1, \ldots, n-1$. These spaces are gathered into the complex $\Omega_{t}^{p, \cdot}(\partial \mathcal{X})$ endowed with differential $\bar{\partial}_{b}$. By the very definition, we get $t(\bar{\partial} f)=\bar{\partial}_{b} t(f)$. The complex $\Omega_{t}^{p, \cdot}(\partial \mathcal{X})$ is usually referred to as the tangential Cauchy-Riemann complex on $\partial \mathcal{X}$, and $\bar{\partial}_{b}$ is called the tangential Cauchy-Riemann operator.

As defined above, the cone of the cochain mapping $t: \Omega^{p, \cdot}(\mathcal{X}) \rightarrow \Omega_{t}^{p, q}(\partial \mathcal{X})$ looks like

$$
0 \longrightarrow \begin{gathered}
\Omega^{p, 0}(\mathcal{X}) \\
\oplus \\
0
\end{gathered} \stackrel{d^{0}}{\longrightarrow} \begin{gathered}
\Omega^{p, 1}(\mathcal{X}) \\
\Omega_{t}^{p, 0}(\partial \mathcal{X})
\end{gathered} \quad \stackrel{d^{1}}{\longrightarrow} \ldots \stackrel{d^{n-1}}{\longrightarrow} \begin{gathered}
\Omega^{p, n}(\mathcal{X}) \\
\Omega_{t}^{p, n-1}(\partial \mathcal{X})
\end{gathered} \longrightarrow 0
$$

where

$$
d^{i}=\left(\begin{array}{rc}
-\bar{\partial}^{i} & 0 \\
t & \bar{\partial}_{b}^{i-1}
\end{array}\right)
$$

Corollary 7.1. Suppose that $\mathcal{X}$ is a compact domain with smooth boundary in a Kähler manifold $\mathcal{X}^{\prime}$ whose interior is $\log \delta$-pseudoconvex. Then the cohomology of (7.1) at steps $0 \leq q \leq n-1$ is zero and the cohomology at the top step $q=n$ is separated.

Note that the latter assertion just amounts to saying that the range of the operator $d^{n-1}$ is closed in $\Omega^{p, n}(\mathcal{X}) \oplus \Omega_{t}^{p, n-1}(\partial \mathcal{X})$.

Acknowledgments The research of the first author was supported by the grant of the Russian Federation Government for scientific research under the supervision of leading scientist at the Siberian Federal University, contract No. 14.Y26.31.0006. 
D. FEDCHENKO AND N. TARKHANOV

\section{REFERENCES}

[AY79] Aizenberg, L. A., and Yuzhakov, A. P., Integral Representations and Residues in Multidimensional Complex Analysis, Nauka, SO, Novosibirsk, 1979.

[AN80] Andreotti, A., and NAcinovich, M., Noncharacteristic hypersurfaces for complexes of differential operators, Ann. Mat. Pura e Appl. 125 (1980), 13-83.

[Bri04] Brinkschulte, J., The $\bar{\partial}$-problem with support conditions on some weakly pseudoconvex domains, Ark. Mat. 42 (2004), 259-282.

[BH08] Brinkschulte, J., and Hill, C. D., On the Cauchy problem for the $\bar{\partial}$ operator, Ark. Mat. 47 (2009), no. 2, 231-241.

[Cal63] Calderón, A. P., Boundary value problems for elliptic equations, In: Outlines of the Joint Soviet-American Symp. on Part. Diff. Eq., Novosibirsk, 1963, 303-304.

[DS96] Dudnikov, P. I., and SAmborski, S. N., Linear overdetermined systems of partial differential equations. Initial and initial-boundary value problems, In: Encyclopaedia of Mathematical Sciences 65, Springer-Verlag, Berlin/Heidelberg, 1996, 1-86.

[Dyn72] Dynin, A., Elliptic boundary problems for pseudo-differential complexes, Funct. Anal. Appl. 6 (1972), no. 1, 75-76.

[HN06] Hill, C. D., and Nacinovich, M., On the failure of the Poincaré lemma for the $\bar{\partial}_{M}$ complex, Part I: Math. Ann. 324 (2002), 213-224; Part II: Math. Ann. 335 (2006), 193-219.

[Kha14] Khavkine, I., The Calabi complex and Killing sheaf cohomology, arXiv: 1409.7212v1 [gr-qc] 25 Sep 2014, 69 pp.

[KTT07] Krupchyk, K., Tarkhanov, N., and Tuomela, J., Elliptic quasicomplexes in Boutet de Monvel algebra, J. Funct. Anal. 247 (2007), no. 1, 202-230.

[KMST05] Kytmanov, A., Myslivets, S., Schulze, B.-W., and Tarkhanov, N., Elliptic problems for the Dolbeault complex, Math. Nachr. 278 (2005), no. 1-2, 194-215.

[MS99] Michel, J., and Shaw, M.-C., $\bar{\partial}$ and $\bar{\partial}_{b}$ problems on nonsmooth domains, In: Proc. of the 40 th Taniguchi Symp. (Katata, 1997), Birkhäuser, Boston, MA, 1999, 159-192.

[SS15] Schulze, B.-W., and SeILER, J., Elliptic Complexes with Generalized Atiyah-PatodiSinger Boundary Conditions, arXiv: 1510.02455v1 [math.AP] 8 Oct 2015, 39 pp.

[ST00] Schulze, B.-W., and TARkhanov, N., A Lefschetz fixed point formula in the relative elliptic theory, Comm. Applied Analysis 4 (2000), no. 1, 99-119.

[See66] SEeley, R. T., Singular integrals and boundary value problems, Amer. J. Math. 88 (1966), 781-809.

[Spa66] Spanier, E. H., Algebraic Topology, McGraw-Hill, New York, 1966.

[Tar90] Tarkhanov, N., A Parametrix Method in the Theory of Differential Complexes, Nauka, SO, Novosibirsk, 1990

[Tar07] Tarkhanov, N., Euler characteristic of Fredholm quasicomplexes, Funct. Anal. and its Appl. 41 (2007), no. 4, 87-93.

[VE65] Vishik, M. I., and Eskin, G. I., Equations in convolution in a bounded domain, Russian Math. Surveys 20 (1965), Issue 3 (123), 89-152.

(Dmitry Fedchenko) Institute of Mathematics and Computer Science, Siberian Federal University, Svobodny Prospekt 79, 660041 Krasnoyarsk, Russia

E-mail address: dfedchenk@gmail.com

(Nikolai Tarkhanov) Institute of Mathematics, University of Potsdam, Karl-LiebkneChtStr. 24/25, 14476 Potsdam (Golm), Germany

E-mail address: tarkhanov@math.uni-potsdam.de 